\title{
Experimental Comparison of Different Scattering Mechanism Selections for Vegetation Height Retrieval by POLINT
}

\author{
J. M. Lopez-Sanchez ${ }^{1}$, J. Fortuny ${ }^{2}$, A. J. Sieber ${ }^{2}$, L. Sagués ${ }^{3}$, M. Bara ${ }^{3}$, X. Fàbregas ${ }^{3}$ and A. Broquetas $^{4}$ \\ ${ }^{1}$ Depto. Física, Ingeniería de Sistemas y Teoría de la Señal, Universidad de Alicante \\ P.O. Box 99, 03080 Alicante, Spain \\ Phone/Fax: +34 965903682, E-mail: juanma@disc.ua.es \\ ${ }^{2}$ Space Applications Institute, JRC, 21020 Ispra (Va), Italy \\ Phone/Fax: +39 0332 785104/5469, E-mail: joaquim.fortuny@jrc.it \\ ${ }^{3}$ Universitat Politècnica de Catalunya (UPC), Campus Nord, c/ Jordi Girona, 1-3, 08034 Barcelona, Spain \\ Phone/Fax: +34 93 4011065/7232, E-mail: sagues@ voltor.upc.es \\ ${ }^{4}$ Institute of Geomatics, Parc de Montjuï, 08038 Barcelona, Spain
}

\begin{abstract}
The application of polarimetric radar interferometry to the estimation of vegetation height is studied in this paper. A comparison between different choices for the scattering mechanisms employed in the retrieval algortihm is presented. When applied to crops, the best results are derived from the height difference between interferograms generated at $\mathrm{HH}+\mathrm{VV}$ and $\mathrm{HH}-\mathrm{VV}$ polarizations.
\end{abstract}

\section{INTRODUCTION}

Polarimetric radar interferometry (POLINT) enables the generation of different interferograms of the same scene. Each interferogram is associated with a scattering mechanism. According to the final application of this technique, the selection of the scattering mechanisms is a critical decision.

This paper is focused on the extraction of vegetation height with POLINT. It is known that parts of trees or plants with different morphological characteristics exhibit different scattering properties. If those parts are physically located at different heights inside the vegetation volume or present dissimilar vertical distributions, their effective phase centers should be placed also at different heights. Since polarimetry can distinguish between scattering behaviors, it can be applied to this problem in order to form interferograms associated with particular scattering mechanisms. In that way, the phase difference between these interferograms is directly related to the height of the vegetation cover in the scene.

This paper discusses some approaches that have been employed so far in the selection of the scattering mechanisms used for these simultaneous interferograms. A direct comparison when applied to crops is also presented, based on experimental data acquired in controlled conditions with ground-based systems.

\section{SELECTION OF SCATTERING MECHANISMS}

Two general methods have been employed so far in the choice of the scattering mechanisms used for these simultaneous inter-

This work has been carried out in the frame of the European Commission TMR network on radar plarimetry ferograms. The first method is based on a recently published algorithm for optimizing the interferometric coherence [1]. This optimization produces three pairs of scattering mechanisms which result in different coherences. In case of a non vegetated surface, all three pairs of scattering mechanisms produce interferograms centered at the surface topography. When we are interested in computing the height of a vegetation volume present in the scene (or an estimate related to the absolute height), we have to calculate the phase difference between first and second, or second and third, or first and third. The interpretation of those scattering mechanisms is not easy. They are selected to maximize the coherence, but no information is provided about their relative position inside the vegetation volume. Each mechanism may be located at the top of the plants, or at the ground level, or somewhere in between. Therefore, we cannot infer which mechanisms should be used for calculating the best parameter related to the actual height of the plants. Some experimental results obtained with this technique were discussed in $[2,3]$.

In contrast with this choice resulting from the coherence optimization, a selection based on the knowledge of the scattering properties of the imaged scene can be employed. It consists in deciding a priori which scattering mechanisms should be used. For example, if one knows that the cross-polar return is mostly produced by the crown of the plants, and that the horizontal polarization backscatter comes from the ground-stem interaction, two interferograms at $\mathrm{HH}$ and $\mathrm{HV}$ should be computed. Evidently, the advantage is the easier interpretation of the scattering mechanisms we are dealing with. The drawback is that it requires a previous knowledge of the scene. Examples of this approach are reported in $[4,5]$. As far as the phase accuracy is concerned, the variance of the relative height differences is quite similar in both approaches, because most of the times the selected scattering mechanisms in the second approach exhibit coherences of the same order as those of the optimization [6]. In this work, both approaches are compared when applied to crops. Two examples of the second approach are presented: a direct use of the three channels provided by the $\mathrm{HV}$ basis $\mathrm{HH}$, 

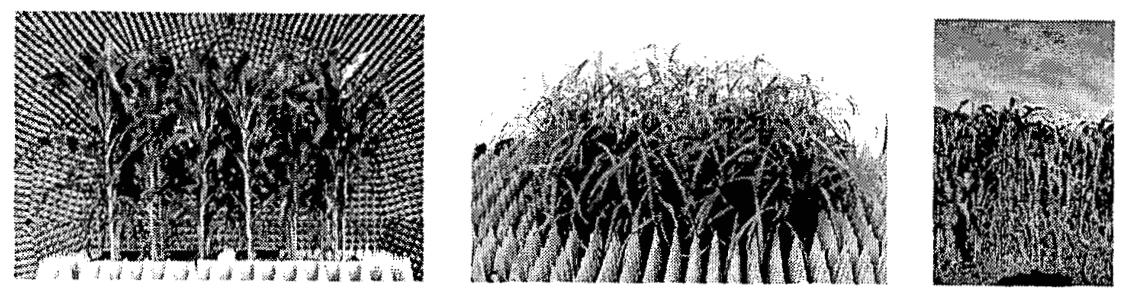

Figure 1: Photographs of crop samples: maize, rice and sorghum

HV and VV), and a choice based on Pauli basis (HH+VV, HHVV and HV).

\section{EXPERIMENTAL RESULTS}

This technique has been tested with two data sets. The first corresponds to maize and rice samples measured at L, S, C and $\mathrm{X}$ band in laboratory conditions at the European Microwave Signature Laboratory, at JRC Ispra, Italy. The second consists of outdoor experiments performed with the fully polarimetric wide-band ground-based scatterometer developed at the Universitat Politècnica de Catalunya (UPC), Barcelona, Spain. Measurements were conducted on bare rough surfaces and two sorghum fields at different growth stages. Fig. 1 shows photographs of all three crops: maize, rice and sorghum.

The maize sample consists of $6 \times 6$ plants about $1.8 \mathrm{~m}$ high. Fig. 2 shows the heights derived for every single scattering mechanism as a function of frequency. The absolute height of a mechanism is known thanks to the EMSL configuration because the ground level is prefectly located. However, the important parameter is not the absolute height with respect to the ground level, but the relative height differences between mechanisms. The best performance is provided by two mechanisms of the Pauli basis: $\mathrm{HH}+\mathrm{VV}$ and HH-VV. HH-VV characterizes a dihedral behavior which corresponds to the interaction of ground and stems. The phase center of this scattering mechanism is ideally close to the ground level [7]. Instead, HH+VV corresponds to direct scattering from the above ground particles, so it is located clearly separate from the ground. These two scattering mechanisms yield the maximum height difference (about $40 \mathrm{~cm}$ ) and behave regularly over the whole frequency range employed in the experiment. Results obtained by using directly the $\mathrm{HV}$ basis are not so well separated. Finally, the mechanisms provided by the coherence optimization are not well separated and behave irregularly with frequency.

Similar comments can be written for the rice sample, which consists of $9 \times 9$ plants about $60 \mathrm{~cm}$ high. At first sight, the estimates behave more irregularly than for the maize sample: only for frequencies highèr than $4 \mathrm{GHz}$ the separation between scattering mechanisms follows the expected distribution (i.e. HH$\mathrm{VV}$ at the bottom and $\mathrm{HH}+\mathrm{VV}$ at the top). At low frequencies there exist many fluctuations on the estimates and they are not well separate. This phenomenon may be produced by differ- ent causes. For example, at low frequencies all the backscatter return is dominated by the ground-stem interaction, which is many dB's above the scattering by the rest of the plant components. Moreover, the physical structure of the plants is more random than maize. As in the maize experiment, the optimum scattering mechanisms vary significantly their relative position inside the volume when the frequency changes. For instance, from 4 to $5 \mathrm{GHz}$ the first is at the bottom and the third at the top, whereas from 6.5 to $7.5 \mathrm{GHz}$ they are swapped. There are also bands with all three optimum mechanisms centered at the same height. Therefore, their choice is not a good starting point for height estimation. In contrast, the mechanisms associated with the Pauli matrices exhibit a fairly uniform separation of about $20 \mathrm{~cm}$ independently from frequency. Regarding the HV option, its estimates are not as clearly separate as the Pauli matrices ones.

The sorghum crops were not homogeneous and their height differed from $1 \mathrm{~m}$ to $1.2 \mathrm{~m}$ in the first field, whereas in the second field the height varied between 1.2 and $1.4 \mathrm{~m}$. The size of the test fields was $10 \times 10 \mathrm{~m}$ and the distance between the antennas and the center of the test field was $18 \mathrm{~m}$, covering an incident angle range from 55 to 65 degrees. Fully polarimetric data were collected at S, C and X band for several baseline distances. Spatial resolutions were about $1.6 \mathrm{~m}$ along ground-range and $0.8 \mathrm{~m}$ along cross-range. In this campaign, the most important source of coherence degradation was temporal decorrelation, mainly caused by the time interval between the acquisitions and the oscillation movement of the plants produced by a strong wind. However, the obtained results confirm the same behavior described in the indoor measurements: the best height estimation is given by the scattering mechanisms of the Pauli basis (HH+VV and HH-VV). Fig. 4 shows the absolute height derived at $\mathrm{C}$ band by selecting different scattering mechanisms along the ground range dimension corresponding to the first sorghum field.

\section{CONCLUSIONS}

Polarimetric SAR interferometry has been tested when applied to crop height estimation. Some experimental results have been obtained with ground-based radars, both in outdoor and indoor operation. The results processed are quite satisfactory. Moreover, a comparison between different choices of scatter- 

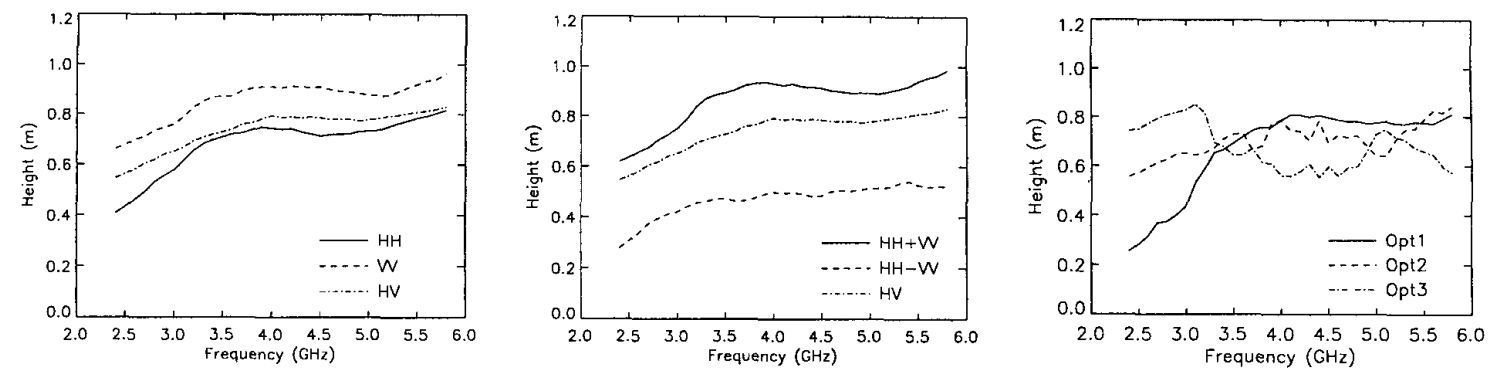

Figure 2: Maize sample. Height of the scattering centers vs frequency. Parameters: $B=0.25^{\circ}, \theta_{i}=45^{\circ}$. Left: HV basis. Center: Pauli matrices. Right: optimum mechanisms.
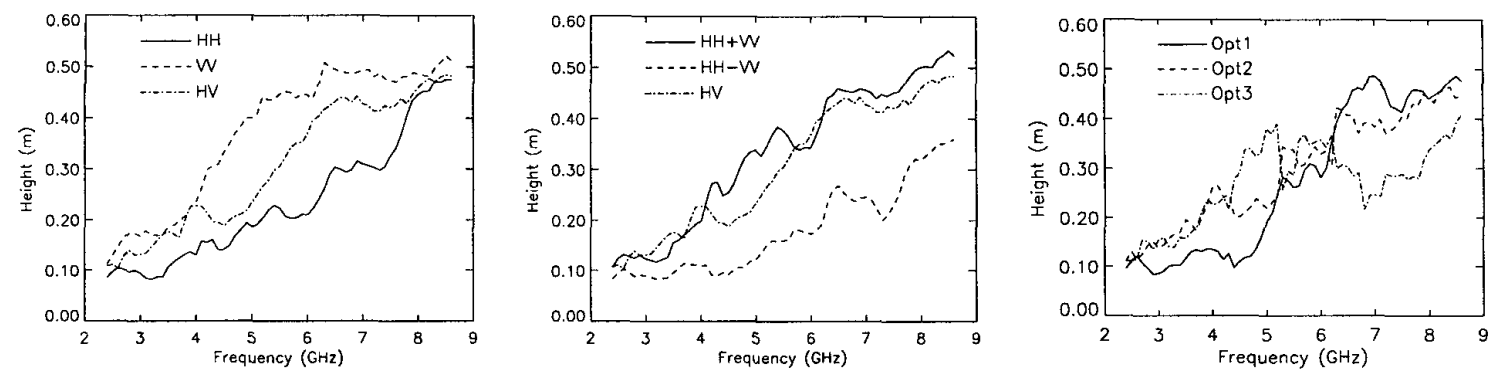

Figure 3: Rice sample. Height of the scattering centers vs frequency. Parameters: $\theta_{i}=45^{\circ}, B=0.5^{\circ}$. Left: HV basis. Center: Pauli matrices. Right: optimum mechanisms.
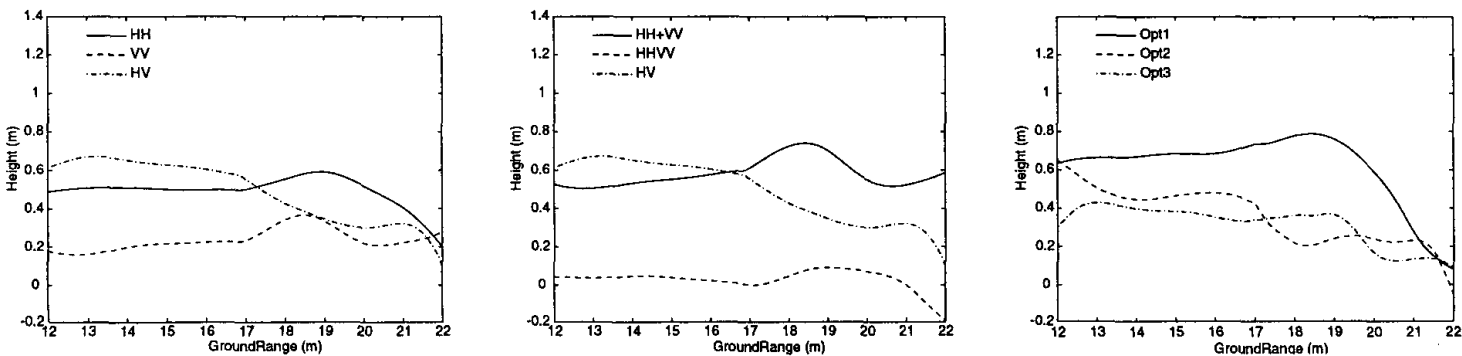

Figure 4: Sorghum field. Height of the scattering centers vs ground range at $\mathrm{C}$ band. Left: HV basis. Center: Pauli matrices. Right: optimum mechanisms.

ing mechanisms to generate the interferograms has been carried out. In general, the best results are provided by the $\mathrm{HH}+\mathrm{VV}$ and HH-VV channels. The first is related to the direct scattering of the above-ground volume, whereas the second is associated with the dihedral response of the ground-stem interaction which is located close to the ground level.

\section{REFERENCES}

[1] S. R. Cloude and K. P. Papathanassiou, "Polarimetric SAR interferometry," IEEE Trans. Geosci. Remote Sensing, vol. 36, pp. 1551-1565, Sept. 1998.

[2] K. P. Papathanassiou and S. R. Cloude, "Phase decomposition in polarimetric SAR interferometry," in Proceedings of the IEEE International Geoscience and Remote Sensing Symposium (IGARSS), Seattle, WA, USA, vol. 4, pp. 2184-2186, July 1998.

[3] J. Gabriel, C. Schmullius, K. P. Papathanassiou, D. Darizhapov, G. Tathkov, T. Tsybjitov, and W.-M. Boerner, "Tree height extraction us- ing polarimetric SAR interferometry," in Proceedings of the IEEE International Geoscience and Remote Sensing Symposium (IGARSS), Hamburg, Germany, vol. 4, pp. 2131-2133, June 1999.

[4] C. B. Wilsen, K. Sarabandi, and Y.-C. Lin, "The effect of tree architecture on the polarimetric and interferometric radar responses," in Proceedings of the IEEE International Geoscience and Remote Sensing Symposium (IGARSS), Seattle, WA, USA, vol. 3, pp. 1499-1501, July 1998.

[5] K. Sarabandi, C. G. Brown, and L. Pierce, "Tree height estimation from the polarimetric and interferometric radar response," in Proceedings of the IEEE International Geoscience and Remote Sensing Symposium (IGARSS), Hamburg, Germany, vol. 4, pp. 1986-1988, June 1999.

[6] J. M. Lopez-Sanchez, Analysis and Estimation of Biophysical Parameters of Vegetation by Radar Polarimetry. $\mathrm{PhD}$ thesis, Polytechnic University of Valencia (UPV), Valencia, Spain, Jan. 2000. .

[7] K. Sarabandi, " $\triangle \mathrm{k}$-radar equivalent to interferometric SAR's: A theoretical study for determination of vegetation height," IEEE Trans. Geosci. Remote Sensing, vol. 35, pp. 1267-1276, Sept. 1997. 